\title{
Non-uniform Frame-rates Improve Single Particle Resolution Data
}

Amy Bondy ${ }^{1}$ and $\mathrm{Min} \mathrm{Su}^{2}$

${ }^{1}$ University of Michigan, Ann Arbor, Michigan, United States, ${ }^{2}$ Life Sciences Institute/University of Michigan, Ann Arbor, Michigan, United States

Beam-induced specimen drift is inherently an issue in single particle cryogenic electron microscopy (cryoEM). ${ }^{1}$ Since the advent of direct electron detectors, motion correction algorithms have sought to correct this drift by collecting movies composed of a number of frames sampled with a uniform frame-rate. ${ }^{2}$ However, initial temporal drift is often substantially higher than later drift, thus uniform frame-rates do not sufficiently sample specimen movement in the early frames and leave redundancy in later frames.

The present study describes a newly developed non-uniform image acquisition scheme. We characterized the average drift per indexed-frame from tilted and zero-degree datasets ( 2000 micrographs per dataset; Figure 1), and clearly observed a trend following exponential decay. To prove the concept, we designed an imaging experiment for a zero-degree single particle dataset with the following steps:

1. Movies with uniform frame lengths of $50 \mathrm{~ms}$ were collected with a dose rate of $\sim 6.0$ electrons $/ \AA^{2} / \mathrm{s}$ and total exposure time of $6000 \mathrm{~ms}$.

2. The same movies were combined by grouping two neighboring frames, resulting in uniform frames of $100 \mathrm{~ms}$ for motion correction.

3. Movies were combined by grouping four neighboring frames, resulting in uniform frames of 200 ms for motion correction.

4. Movies were generated with uniform frame lengths of $50 \mathrm{~ms}$ for the first $200 \mathrm{~ms}$ followed by uniform (grouped) frames lengths of $100 \mathrm{~ms}$ for the second $800 \mathrm{~ms}$, and ended with uniform (grouped) frames of $200 \mathrm{~ms}$ for the last $5000 \mathrm{~ms}$. The resulting hybrid movies were subjected to motion correction.

Example drift plots from these four different scenarios are depicted in Figure 2a. The non-uniform framerate movie captures the best features of all three uniform frame-rate schemes. Following motion correction, contrast transfer function (CTF) estimation was performed for the four different scenarios described above. ${ }^{3}$ As the largest differences between the imaging schemes occurred within the first 1000 $\mathrm{ms}$, we focused on the CTF of these initial frames. In Figure $2 b$ the CTF amplitude and quality of fit curves are shown for the $50 \mathrm{~ms}$ uniform frame length, $200 \mathrm{~ms}$ uniform frame length, and non-uniform frame length scenarios. At higher resolution $(\sim 5 \AA)$ the non-uniform scheme has a better CTF fit than the $200 \mathrm{~ms}$ uniform scheme, suggesting an improved signal to noise ratio. The non-uniform scheme also has individual movie files $30 \%$ the size of the $50 \mathrm{~ms}$ uniform scheme since much fewer frames are needed to achieve a comparable resolution. These findings suggest that using a non-uniform frame length acquisition scheme may lead to improved resolution in 3D reconstruction maps, particularly for tilted single particle data, without additional computational storage needed or complex processing. 


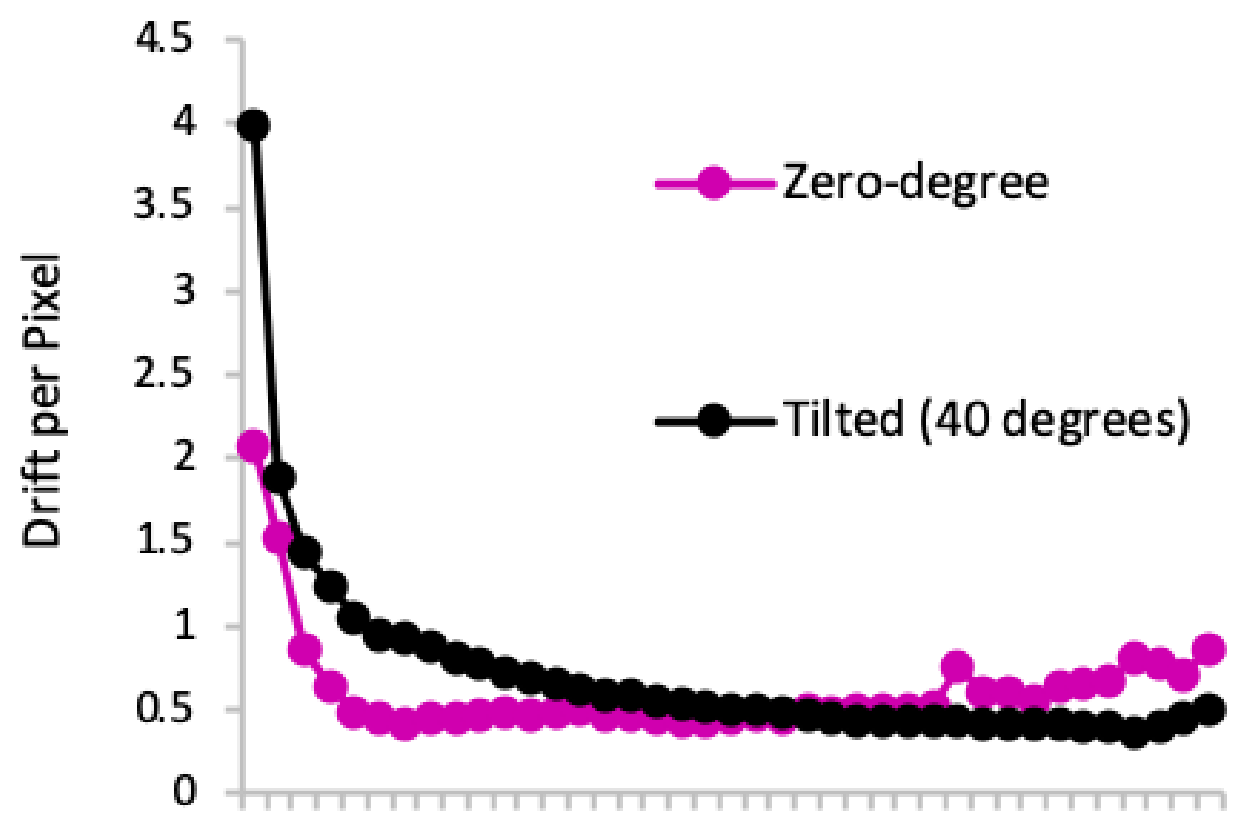

\section{9}

\section{Frame Number}

Figure 1. Average drift per indexed-frame for an entire tilted and zero-degree dataset.
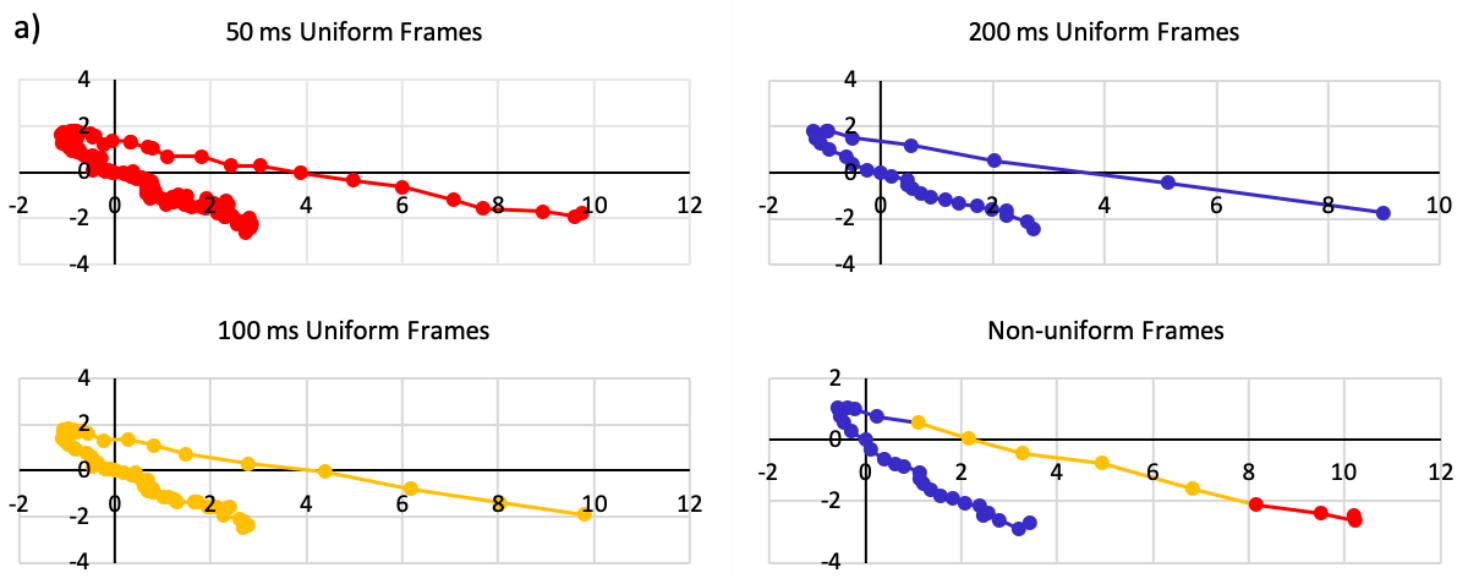

b)

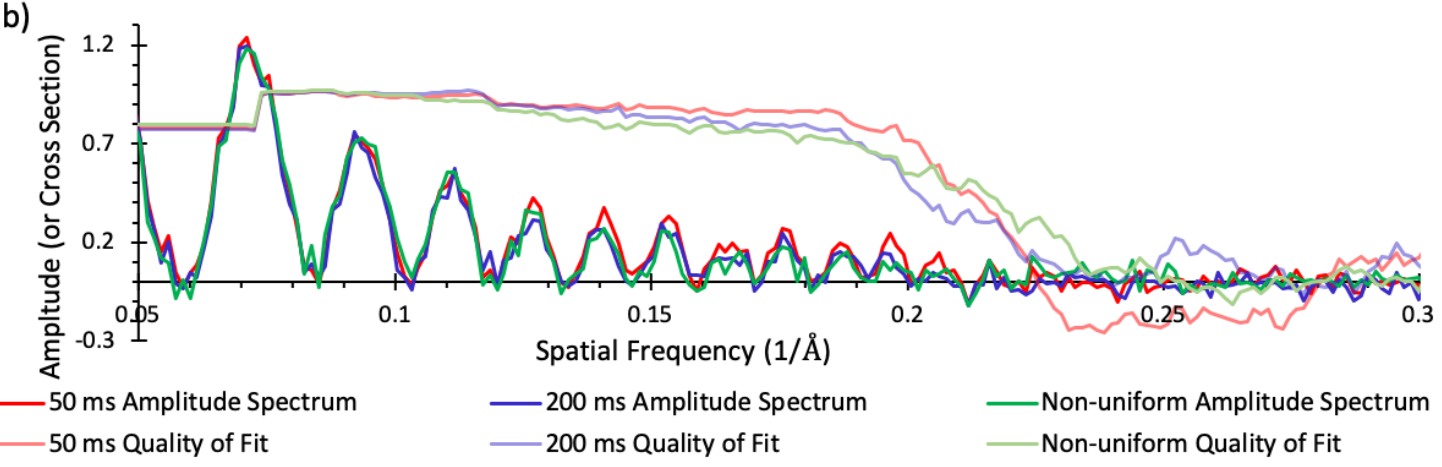

Figure 2. Figure 2. (a) Example drift plots of a movie denoting drift in units of pixels for $50 \mathrm{~ms}$ uniform length frames (red), $100 \mathrm{~ms}$ uniform length frames (yellow), and $200 \mathrm{~ms}$ uniform length frames (blue). 
The non-uniform frame-rate drift plot denotes each segment (red, yellow, and blue) based on the its respective frame length. (b) CTF amplitude and quality of fit for micrographs collected with $50 \mathrm{~ms}$ uniform length frames (red), $200 \mathrm{~ms}$ uniform length frames (blue), and a non-uniform frame length scheme (green).

\section{References}

1. D Lyumkis, J. Biol. Chem. 294 (2019), p. 5181.

2. X Li, P Mooney, S Zheng, CR Booth, MB Braunfeld, S Gubbens, DA Agard, Y Cheng, Nat. Methods 10 (2013), p. 584.

3. A Rohou and N Grigorieff, J. Struct. Biol. 192 (2015), p. 216. 
https://doi.org/10.1017/S1431927620021133 Published online by Cambridge University Press 